\title{
Is Volatility Clustering of Asset Returns Asymmetric?
}

\author{
Cathy Ning*a ${ }^{* a}$ Dinghai $\mathrm{Xu}^{b}$, and Tony S. Wirjanto ${ }^{c}$ \\ ${ }^{a}$ Department of Economics, Ryerson University,Toronto, Ontario, Canada, M5B 2K3. \\ ${ }^{b}$ Department of Economics, University of Waterloo, Waterloo, Ontario, Canada, N2L 3G1. \\ ${ }^{c}$ School of Accounting \& Finance and Department of Statistics \& Actuarial Science, \\ University of Waterloo, Waterloo, Ontario, Canada, N2L 3G1. \\ This Version: March 2014
}

\footnotetext{
${ }^{*}$ Corresponding author: Tel.: 1-416-979-5000 ext. 6181; Fax: 1-416-598-5916; E-mail address: qning@ryerson.ca. Cathy Ning gratefully acknowledges the financial support from the Social Sciences and Humanities Research Council of Canada (SSHRC) grant \#118746. Earlier versions of the paper were circulated under the title "Modeling Asymmetric Volatility Clusters with Copulas and High Frequency Data". We thank participants at the 2013 Conference on Copulas and dependence: Theory and Applications in Columbia University, 2013 Asian Meeting of the Econometric Society in Singapore, the 2011 Annual Conference of the Canadian Economics Association in Ottawa, and 2011 Annual Meeting of the Canadian Econometrics Study Group in Toronto for useful comments and suggestions on the earlier versions of the paper.
} 


\begin{abstract}
Volatility clustering is a well-known stylized feature of financial asset returns. In this paper, we examine whether the volatility clusters are symmetric by focusing our investigation on the structure of the clusters of volatilities in both the stock and foreign exchange markets. The employment of copula-based semi-parametric univariate timeseries models accommodates the clusters of both high and low volatilities of returns in the analysis. Using daily realized kernel volatilities constructed from high frequency return data, we find that volatility clustering is highly nonlinear and strongly asymmetric: that is, clusters of high volatilities tend to be much more pronounced than clusters of low volatilities. Our paper is the first one to address and document the asymmetry of volatility clustering. The finding is consistent with the asymmetric leverage effect and volatility feedback effect documented in recent studies. In addition, the volatility clusters are shown to remain persistent and asymmetric even after forty days. This finding is consistent with the long memory dependence in volatility documented in the literature.
\end{abstract}

Keywords: Volatility clustering, Univariate time series copulas, Realized kernel volatility, High-frequency data.

JEL Classification: C51, G32. 


\section{Introduction}

One of the best known stylized features of financial asset returns is volatility clustering. That is, high volatility movements (represented by large changes in returns) are observed to be followed by high volatility movements (characterized by a period of relative market turbulence), while low volatility movements (indicated by returns hardly fluctuating) are followed by low volatility movements (corresponding to a period of a relative tranquil market). The former signifies the clusters of high volatility while the later signifies the clusters of low volatility. This pattern of volatility clustering captures the dynamics of volatility fluctuations in financial assets. Modeling this type of volatility clustering of financial market is clearly important since volatility values can directly impact prices of options and risks of stocks and portfolios. So far and to the best of our knowledge, volatility clustering has mainly been analyzed within the class of Generalized Autoregressive Conditional Heteroscedastic (GARCH) and Stochastic Volatility (SV) models. Each class of these models can well capture the symmetric volatility clustering pattern in asset returns, i.e., the clusters of high volatilities and low volatilities are modeled in a symmetric way. However, an important research question arises as to whether volatilities actually cluster the same way during turbulent times and tranquil times in the markets? In fact we often observe turbulent market periods occur more frequently than tranquil market periods; that is, high volatilities of returns tend to cluster more often than low volatilities of returns. We call this phenomenon of asymmetry in the frequency of clusters of high volatilities and low volatilities än asymmetric volatility clustering: The GARCH and SV models are not equipped to capture this type of asymmetry 
in volatility clustering. This paper models this type of possible asymmetric and non-linear volatility clusters and examines them empirically.

In this paper, we contribute to the literature by investigating the structure of volatility clustering of financial asset returns by using a copula approach. Copulas allow us to capture the structure of the clusters of the volatilities, of either a symmetric or an asymmetric type. It is well known that copula is a multivariate distribution function of the standard uniform marginals. In particular, due to Sklar's theorem, the dependence between variables can be modeled separately via certain copula functions and their corresponding marginals. Further, a copula approach can accommodate various types of marginal distributions. Moreover, different copulas are designed to capture different types of dependence structures: symmetric or asymmetric, and linear or nonlinear. This property of copulas provides flexibility in the modeling of dependence in the return data.

Due to this flexibility, the copula approach has been extensively applied in Finance in recent years. Just to mention a few, Jondeau and Rockinger (2006) use both the conditional and dynamic copula models to examine the dynamic dependence of the US and European stock market returns. Rodriguez (2006) investigates market contagions via copulas. Patton (2006) employs copulas to model the asymmetric dependence on the foreign exchange rate markets. More recently, Chollete et al. (2011) use copula approaches and find asymmetric extreme dependence among equity returns, which indicates that the stock markets tend to crash together but do not boom together. Ning (2010) and Wang et. al. (2013) apply copulas to examine nonlinear dependence between stock and foreign exchange markets. Chu (2011), 
Boubaker and Sghaierand (2013), and Low et. al. (2013) emphasize the advantages of using copulas in asset allocation and portfolio optimization. Lastly a survey on applications of copulas in finance and economics can be found in Patton (2009).

While most applications of the copula approach are in the multivariate context, Chen and Fan (2006) develop theoretical foundations for extending the copula approach to model dependence of a univariate variable across time. Following Chen and Fan (2006), we apply the copula approach to a single time series variable and employ a semi-parametric estimation method to study the pattern of volatility clustering in financial asset returns. The advantage of this method is that we do not need to specify the marginal distribution of the volatility; instead, we estimate the volatility with an empirical distribution function that is distribution-free. In this paper we focus on the conditional volatility clustering, which, more precisely stated, is the dependence between consecutive volatilities; that is, given the volatility level in the previous period we are interested in the question of how often does current volatility clusters with previous volatility using a number of parametric copulas. The copula parameters are estimated by a two-step maximum likelihood method, also known as the Canonical Maximum Likelihood (CLM) method.

We note that copulas possess a number of attractive properties for the purpose of studying volatility dependence. First, copulas are designed to capture both nonlinear and linear dependence in a time series. This is especially important in the investigation of the dependence of the volatility clusters, which may not be linear. Second, copulas can accommodate any types of continuous marginal distributions, including a family of skewed and fat-tailed 
distributions, which can be used to characterize our volatility data with significant positive skewness coefficients and large excess kurtosis values. Third, copulas are invariant to a strictly increasing transformation, including a nonlinear transformation. This feature is especially convenient since very often our transformation of the data is increasing but nonlinear. For further properties of copulas, see, for instance, Joe (1997), Nelson (1999), Embrechts et al. (2002), and Cherubini et al. (2004).

In this paper, the characteristics of volatility clustering are examined for the returns from both the stock and foreign exchange rate markets. We construct daily realized volatilities using high frequency data. We find that, in our sample data, volatility clustering is mostly asymmetric, in the sense that volatility clustering occurs more often for high volatilities than for low volatilities. This asymmetric pattern also exists in the dynamics of volatility clusters. That is, high volatilities, not only on average, but also across time, cluster more often than low volatilities. This result reveals that, in addition to the volatility clustering found previously in the GARCH and SV models, the structure of the volatility clustering exhibits asymmetric patterns, with an increasing tendency for clusters for high volatilities to occur relative to low volatilities. This, to the best of our knowledge, has not been documented in the literature so far. This new result implies that consecutively highly volatile periods tend to occur more often than consecutively tranquil periods in both the stock and currency markets.

Our finding of asymmetric volatility clusters is consistent with the asymmetric leverage effect and volatility feedback effect documented in recent studies. Notably, Bollerslev et. 
al. (2006) find a highly significant, prolonged leverage effect (i.e., volatility increases more following negative returns than positive returns.) and an almost instantaneous volatility feedback effect (i.e., an increase in volatility results in negative returns.) for the intra-daily data. In particular, our findings suggest that, through the leverage effect, a negative return initially amplifies the extent of subsequent volatility, and this, through the volatility feedback effect, induces a negative return. This negative return, in turn, through the leverage effect, intensifies the degree of the subsequent volatility. The tendency for these two effects to be prolonged and asymmetric implies a prolonged clustering of large volatilities.

In addition, in this paper, we also investigate the duration of the volatility clustering in the return series. Our results indicate that the clusters in most of the volatilities of the return series examined in this paper tend to be highly persistent and do not appear to dissipate even after forty days. This finding supports the long memory dependence in volatility of the returns as documented in the literature, see Engle and Bollerslev (1986), Bollerslev et al. (1992), Ray and Tsay (2000) and among others.

The rest of the paper is organized as follows. Section 2 presents the copula models for the volatility clustering. Section 3 describes the sample data and the construction of the kernel volatility measures from the high frequency data of the returns. Section 4 presents and discusses the empirical results and Section 5 concludes the paper. 


\section{The Methodology}

Copula is a multivariate cumulative distribution function with the marginal distribution being uniform on the interval $[0,1]$. In this paper, we apply a class of parametric copulas in modeling the dependence between consecutive volatilities of the return series. According to Sklar's (1959) theorem, there is a one-to-one relationship between a joint distribution and a copula. Chen and Fan (2006) extend this property from the multivariate context to a univariate set-up. Let $Y_{t}$ and $Y_{t-1}$ be the consecutive volatility variables at time $t$ and $t-1$ respectively. Then the clustering property of consecutive volatilities is completely characterized by their joint distribution, $H\left(y_{t}, y_{t-1}\right)$. In particular, based on Sklar's theorem, there exists a copula $\mathrm{C}($.$) such that$

$$
H\left(y_{t}, y_{t-1}\right)=C\left(u_{1}, u_{2}, \theta_{c}\right)
$$

where $u_{1}=G\left(y_{t}\right)$ and $u_{2}=G\left(y_{t-1}\right)$ are the marginal cumulative distribution functions of $Y_{t}$ and $Y_{t-1}$, respectively and $\theta_{c}$ is the copula parameter vector. In other words, the copula function is a joint distribution function of the transformed random variables $u_{1}=$

$G\left(y_{t}\right)$ and $u_{2}=G\left(y_{t-1}\right)$. One distinct advantage of copula approach is that it can separate the dependence from marginals, with the dependence completely captured in the copula function. Since our focus is on the dependence between two consecutive volatilities, rather than their marginals, we specify the copula function parametrically (but not the marginal distribution of the volatility). As an important consequence, this particular approach is free of any specification errors of the marginals. This advantageous feature is highlighted in the simulation studies in Fermanian and Scaillet (2005). In this paper, we follow Chen and 
Fan (2006), which extends the method in Genest et al. (1995) from the i.i.d. multivariate time series case to the dependent univariate time series case, and adotp a semi-parametric estimation method (CML). See also Cherubini et al. (2004)) and Joe (1997). In essence, this is a two-step procedure. In the first step, the marginal distribution function $G($.$) is estimated$ non-parametrically via its re-scaled empirical cumulative distribution function (ECDF)

$$
\widehat{G}\left(y_{t}\right)=\frac{1}{T+1} \sum_{t=1}^{T} 1\left\{Y_{t}<y\right\} .
$$

The ECDF is re-scaled to ensure that the first-order condition of the copula's log-likelihood function is well defined for all finite $T .{ }^{1}$ By the Glivenko-Cantelli theorem, $\widehat{G}_{Y}\left(y_{t}\right)$ converges to its theoretical counterpart $G\left(y_{t}\right)$ uniformly. In the second step, given the nonparametrically estimated ECDF, $\widehat{G}\left(y_{t}\right)$ and $\widehat{G}\left(y_{t-1}\right)$, we can estimate the copula parameters $\theta_{c}$ parametrically by the method of maximum likelihood, with

$$
\begin{aligned}
\widehat{\theta}_{c} & =\arg \max _{\theta_{c}} \widetilde{L} \\
\text { where } \widetilde{L}\left(\theta_{c}\right) & =\frac{1}{T} \sum \log c\left(\widehat{G}\left(y_{t}\right), \widehat{G}\left(y_{t-1}\right) ; \theta_{c}\right),
\end{aligned}
$$

where c(.) is the copula density function. Joe (1997) proves that under a set of regularity conditions, the two-step estimator is consistent and asymptotically normal. It is also pointed out that the two-step method is efficient. In addition, as indicated in Patton (2008), this method is computationally tractable. Importantly Chen and Fan (2006) establish the asymptotic properties for the resulting semi-parametric estimator.

Using copulas, we can measure the dependence at the extremes by estimating the extent of the tail dependence. A tail dependence measures the probability that both variables are

\footnotetext{
${ }^{1}$ See Genest et al. (1995) and Chen and Fan (2006) for a further discussion on this.
} 
in their lower or upper joint tails. Intuitively, an upper (lower) tail dependence refers to the relative amount of mass in the upper (lower) quantile of their joint distribution. The lower (left) and upper (right) tail dependence coefficients, $\lambda_{l}$ and $\lambda_{r}$, in the context of volatility dependence (clusters) are defined as:

$$
\begin{gathered}
\lambda_{l}=\lim _{u \longrightarrow 0} \operatorname{Pr}\left[G\left(y_{t}\right) \leq u \mid G\left(y_{t-1}\right) \leq u\right]=\lim _{u \longrightarrow 0} \frac{C(u, u)}{u}, \\
\lambda_{r}=\lim _{u \longrightarrow 1} \operatorname{Pr}\left[G\left(y_{t}\right) \geq u \mid G\left(y_{t-1}\right) \geq u\right]=\lim _{u \longrightarrow 1} \frac{1-2 u+C(u, u)}{1-u},
\end{gathered}
$$

respectively, where $\lambda_{l}$ and $\lambda_{r} \in[0,1]$. If $\lambda_{l}$ or $\lambda_{r}$ is positive, $Y_{t}$ and $Y_{t-1}$ are said to be left (lower) or right (upper) tail dependent. That is, $\lambda_{l}$ measures the degree of clusters of low volatilities, while $\lambda_{r}$ measures the degree of clusters of high volatilities. Since tail dependence measures are derived from the copula functions, they possess the desirable properties of copulas mentioned earlier.

Different copulas represent different dependence structures. We will focus on five copulas that represent popular types of dependence structures in finance: Gaussian copula, Student T copula, Clayton copula, Clayton survival copula, and the Symmetrized Joe Clayton (SJC) copula.

The Gaussian copula serves as a natural benchmark, as it does not have tail dependence and is widely assumed in financial models. The bivariate Gaussian copula has the form as follows:

$$
C\left(u_{1}, u_{2} ; \rho\right)=\Phi_{\rho}\left(\Phi^{-1}\left(u_{1}\right), \Phi^{-1}\left(u_{2}\right), \rho\right),
$$

where $\Phi_{\rho}$ is a bivariate normal distribution function with the correlation coefficient $\rho$, and $\Phi^{-1}$ is an inverse of the univariate normal distribution function. This copula is determined 
by only one parameter $\rho$ and $\lambda_{l}=\lambda_{r}=0$. In terms of volatility clustering, a Gaussian copula implies no clusters of very high or low volatilities.

The $t$ copula is defined as

$$
C_{v, \rho}\left(u_{1}, u_{2}\right)=t_{v, \rho}\left(t_{v}^{-1}\left(u_{1}\right), t_{v}^{-1}\left(u_{2}\right)\right)
$$

where $t_{v, \rho}$ is a bivariate student $\mathrm{t}$ distribution with degrees of freedom (DoF) denoted by $v$ and the correlation coefficient $\rho . t_{v}^{-1}$ is an inverse of the univariate student $\mathrm{t}$ distribution. The advantage of a $\mathrm{T}$ copula is that it retains a linear correlation measure $\rho$ and in addition to that, it introduces a parameter, DoF $v$, that determines the extent of symmetric tail dependence. It also nests the Gaussian copula. In the T copula, clusters of both high and low volatilities are permitted, but the occurrence of clusters is equally likely.

The Clayton copula is defined as:

$$
C\left(u_{1}, u_{2}\right)=\left(u_{1}^{-\alpha}+u_{2}^{-\alpha}-1\right)^{1 / \alpha} \text { for } \alpha>0
$$

where $\alpha$ is an associate parameter. Different from the $\mathrm{T}$ copula, the Clayton copula allows only a lower tail dependence: $\lambda_{l}=2^{-1 / \alpha}, \lambda_{r}=0$. Therefore a Clayton copula is designed to capture clusters of only low volatilities but not high volatilities.

The Clayton survival copula can be expressed as

$$
C_{\text {Sur }}\left(u_{1}, u_{2}\right)=\left[\left(1-u_{1}\right)^{-\alpha}+\left(1-u_{2}\right)^{-\alpha}\right]^{1 / \alpha}+u_{1}+u_{2}-1 \quad \text { for } \alpha>0
$$

In contrast to the Clayton copula, the Clayton survival copula allows for only upper tail dependence: $\lambda_{l}=0, \lambda_{r}=2^{-1 / \alpha}$. This implies that only clusters of very high volatilities, but not very low volatilities, are captured in the Clayton survival copula. 
Finally, the SJC copula is defined as

$$
C_{S J C}\left(u_{1}, u_{2} \mid \lambda_{r}, \lambda_{l}\right)=0.5 \times\left(C_{J C}\left(u_{1}, u_{2} \mid \lambda_{r}, \lambda_{l}\right)+C_{J C}\left(1-u_{1}, 1-u_{2} \mid \lambda_{l}, \lambda_{r}\right)+u_{1}+u_{2}-1\right),
$$

where $C_{J C}\left(u, v \mid \lambda_{r}, \lambda_{l}\right)$ is the BB7 copula (also called Joe-Clayton copula) of Joe (1997) defined as

$$
\begin{aligned}
& C_{J C}\left(u_{1}, u_{2} \mid \lambda_{r}, \lambda_{l}\right) \\
= & 1-\left(1-\left\{\left[1-\left(1-u_{1}\right)^{k}\right]^{-r}+\left[1-\left(1-u_{2}\right)^{k}\right]^{-r}-1\right\}^{-1 / r}\right)^{1 / k},
\end{aligned}
$$

where $k=1 / \log _{2}\left(2-\lambda_{r}\right), \quad r=-1 / \log _{2}\left(\lambda_{l}\right)$, and $\lambda_{l} \in(0,1), \lambda_{r} \in(0,1)$. The SJC copula allows for an asymmetric upper and lower tail dependence and by construction, it is symmetric when $\lambda_{l}=\lambda_{r}$ as a special case. A SJC copula captures clusters of both high and low volatilities and they are not necessarily symmetric. Thus this is, by far, the most flexible copula of all of the copulas studied in this paper.

In summary, the above five copulas capture the most important types of dependence for financial data. We use AIC and BIC to assess and compare the performance of these copula models.

\section{Data Description and Construction of Realized Mea- sure for Volatility}

It is well-known that volatility can not be directly observed in the financial markets. In this paper, a popular model-free measure for volatility is used in the analysis, namely realized 
kernels. Asymmetric volatility clusters are examined for both the stock and foreign exchange rate (FX) markets. Our sample data set consists of five stock market indices, including the S\&P 500 (SPX), Russell 2000 (RUT), Dow Jones Industrial Average (DJI), Nasdaq 100 (IXIC) and EURO STOXX 50 index (SToXX50) for the period January 2, 2002-April 10.,2012 and four foreign exchange rates, including Euro (EUR), Great British pound (GBP), Switzerland Franc (CHF) and Japanese yen (JPY) from January 31999 to March 1, 2009. All the currencies are in terms of the US dollars. The sample interval covers different periods dictated by the data availability. ${ }^{2}$

A standard model-free indicator of the daily volatility is a simple summation of squared intra-daily returns, see Andersen and Bollerslev (1998), Barndorff-Nielsen and Shephard (2001) and etc. To set forth the notation, let $p_{d, t}$ be the logarithmic price at a certain sampling frequency interval on day $t$. Consequently, the continuously compounded returns with $D$ observations on day $t$ is defined as $r_{d, t}=100\left(p_{d, t}-p_{d-1, t}\right)$, where $d=1,2, \ldots, D$ and $t=1,2, \ldots, T$. When $d=1$, the first subscript is ignored and $r_{t}$ denotes the return series on a given day. A simple estimator of the daily volatility can be constructed by summing up the squared intra-day returns when the market is open, i.e., $R V_{t}=\sum_{d=1}^{D} r_{d, t}^{2}$. Under the assumptions that returns have zero mean, zero correlation and finite second moments, $R V$ is a consistent measure of the daily volatility under an ideal market condition. However, in practice, there are several potential issues in the above construction for the realized volatility. We elaborate them below.

\footnotetext{
${ }^{2}$ Our data source are from www.tickplusdata.com and realized library of Oxford-man Institute of Quantitative Finance.
} 
Due to a rapid development in the computer technology, the financial transaction data have become available at the ultra high frequency level. In particular, the transaction price nowadays can be recorded at a very fine time scale, such as transaction by transaction (tickby-tick), one minute, five minutes and etc. This raises the question as to which set of discrete time series data we should choose as an appropriate measure of the realized volatility. The choice of the sampling frequency is not trivial by any means. Intuitively, a larger data set should always contain more information. As the sampling interval approaches zero, the realized volatility can be equivalently considered as an integrated estimator. However, there is an important trade-off involved in choosing the observation frequency due to the presence of the market microstructure noises. In other words, financial asset prices diverge from their "efficient values" due to a variety of market frictions. Recently, increasing attention has been focused on the analysis of the realized volatility measures, see for instance Zhang et al. (2005), Hansen and Lunde (2006), van Dijk and Martens (2007), Andersen, Bollerslev and Diebold (2007), Maheu and McCurdy (2009) and etc. See also a survey paper on this topic by McAleer and Medeiros (2008). ${ }^{3}$

Another non-trivial issue in the construction of RV is the noise contamination effects. There are several typical sources of noise embedded in the high frequency trading data.

\footnotetext{
${ }^{3}$ In the original version of the paper, we also have the results for the realized volatility. But, to save space, the results are not reported here. To construct the RV, we follow most of the literature and simply use 5minute sampling intra-day data because this interval is considered as the frequency at which the transaction prices are less distorted by the microstructure noises. Those results are available upon request from the authors.
} 
For example, Hasbrouck (1993) mentions that noise could arise from discreteness, noninformation based component of bid-ask spread, etc. In the presence of this noise, realized volatility could be a biased estimator as shown in Barndorff-Nielsen and Shephard (2002). For this reason, the popular realized kernel is adopted in the paper. The kernel estimator for volatility was first introduced by Barndorff-Nielsen et al. (2008). Unlike the realized volatility, Barndorff-Nielsen et al. $(2008,2009)$ show that the proposed realized kernel estimator is a robust estimator for the underlying integrated volatility even when the high frequency trading data are contaminated with noises. Their empirical results also show that the realized kernel provides a better performance than the traditional realized volatility measure. Following Barndorff-Nielsen et al. (2008, 2009), in this paper, our realized kernel estimator has the form of equation (1.2) in Barndorff-Nielsen et al. (2009). According to Sheppard (2008), the nonflat-parzen kernel function is used on the data at the tick-by-tick level.

The summary statistics for the volatilities of the returns are presented in Table 1. In particular, the first four moments are reported. In general, the mean and variance of the volatilities in the FX market are observed uniformly smaller than those from the stock markets, reflecting much greater volatility in the stock markets than in the foreign exchange markets. We could also see this phenomenon from our range statistics. We note that the constructed realized kernel volatilities are all skewed to the right, indicating a longer right tail. The kurtosis coefficients range from 44.2 to 301.2 , showing an extreme fat-tail property of the distributions. Both the skewness and kurtosis coefficients indicate that the volatility series are far away from a normal distribution. Thus we cannot use a linear correlation 
method to measure the dependence of such non-normally distributed variables. The copula approach, on the other hand, which allows for any continuous marginal distributions, is expected to provide a more accurate measure for this type of dependence in the data.

Figure 1 presents all of the kernel volatility series. The graph also shows some evidence of clusters of volatilities.

\section{Empirical Results}

In this section, we discuss the results obtained from applying the approach described in section 2 to our kernel volatility series. First, we consider the linear correlation measures of the consecutive volatilities in the first row of Table 2. The reported correlation coefficients range from 0.64 to 0.88 , reflecting a modest to high linear dependence between the consecutive volatilities of the return series. This represents a stylized feature of the volatility clustering of the returns in both the stock and foreign exchange rate markets.

Next, we examine the dependence structure between volatility $Y_{t}$ at time $t$ and $Y_{t-1}$ at time $t-1$ via a set of copula models that represent different dependence structures. The results are presented in Table 2. First, looking at the estimates of dependence parameters in the copula models are all statistically significant, which gives strong evidence for the existence of volatility clustering. Comparing the Gaussian copula and the T copula, we find that the $\mathrm{T}$ copula performs comparatively better with smaller AIC and BIC for all stock indices and exchange rates. That is, the measure which includes tail dependence works better than the measure which does not allow for tail dependence. This is taken as the evidence of 
tail dependence between the consecutive volatilities. Second, comparing the T copula with the SJC copula, we find that the SJC copula dominates the T copula as indicated by much lower AIC and BIC values. This leads us to conclude that the model with asymmetric tail dependence outperforms the model with symmetric tail dependence. Finally, comparing the SJC copula with the Clayton survival copula and the Clayton copula, we find that the SJC copula outperforms both the Clayton survival copula and the Clayton copula, where the latter two allow for only one side tail dependence, either right tail only or left tail only. In summary, based on both the AIC and BIC, the SJC copula is preferred over all other four copulas considered in this paper. This is the evidence of the presence of dependence in both tails.

Next, we focus on the results from the best performed copula, which is the SJC copula. Both the left and right tail dependence parameters are highly statistically significant, indicating the existence of both the left and right tail dependence of the consecutive volatilities. This points to the likelihood of the clusters of low volatilities with low volatilities, and the likelihood of high volatilities with high volatilities. Second, for each asset, the left tail dependence parameter is always smaller than the right tail dependence parameter. This suggests the probability of the clusters of low volatilities is smaller than the probability of the clusters of high volatilities. In other words, high volatilities tend to cluster more often than low volatilities of the return series considered in this paper. This result is also supported by the superior performance of the Clayton survival copula with large upper tail dependence compared to the Clayton copula with smaller lower tail dependence. The asym- 
metric tail dependence reveals that, in addition to the volatility clustering previously found in the GARCH and SV models, volatility clustering is highly asymmetric, with a stronger tendency of clusters of high volatilities than low volatilities. To the best of our knowledge, this result has not been documented in the literature. The calculated t-statistics for the null hypothesis that the left tail dependence parameter is equal to or greater than the right tail dependence against the alternative hypothesis that the left tail dependence parameter is smaller than the right tail dependence is strongly rejected in all cases as indicated by the uniformly small p-values. We interpret this result as evidence that the left tail dependence parameter is significantly smaller than the right tail dependence parameter. Therefore, the volatility clustering is found to be highly asymmetric, i.e., there is a significantly stronger tendency for clusters of large volatilities to occur than small volatilities in the financial asset return series considered in this paper.

The asymmetry in the volatility clustering is consistent with the leverage effect and the return-volatility feedback effect documented in the literature, notably in Bollerslev et al. (2006) for intra-daily volatilities. First, through the leverage effect, a negative return is more likely to be followed by a higher volatility than a positive return. Second through the volatility feedback effect, the higher volatility is likely to be accompanied by a negative return, which, in turn, again through the leverage effect, is followed by another high volatility. Such a process points to clusters of large volatilities. On the other hand, the leverage effect also suggests that a positive return is less likely to be followed by an increase of volatility, indicating a weaker tendency of clusters of small volatilities. 
Interestingly, when comparing the results across different groups of assets, we find that the asymmetric pattern of volatility clusters tends to be more pronounced for the stocks than the exchange rates. We surmise that the stock markets tend to have more extreme events than the exchange rate markets.

It is well documented that the volatility clustering at the daily or weekly interval is strongly persistent and has a long memory, See Bollerslev et al. (1992) for a survey of this literature for ARCH models and also in Ray \& Tsay (2000). Thus it would also be interesting to investigate how long the memory lasts and how slowly the volatility clusters die out. To do this, we examine the clusters of volatilities of the return series from the first lag to the $60 t h$ lag using the SJC copula. We present the results in Table 3. From the table, we first observe that the degree of volatility clusters decreases gradually as the lag increases. This is reflected in generally smaller tail dependence parameters and larger values of the AIC and BIC with the increase of the lag. Second, the decay of the upper tail dependence is much slower than the lower tail dependence, meaning the clusters of the high volatilities do not disappear as fast as the clusters of low volatilities. Low volatility clusters decay rapidly to 0 for all US stock indices and GBP and JPY. Third even after 40 days, the clusters in high volatilities are still both statistically and economically significant in most of the cases, indicating a strong persistence in the clusters of high volatilities. Finally, the clusters decay at a slower speed for the exchange rate volatilities than for the stock indices. Thus volatility clusters are more persistent in the foreign exchange markets than in the stock markets.

To visualize the decaying patterns of the clusters, we plot the left and right tail depen- 
dence parameters against the lags for each group in Figures 2 and 3. These figures confirm visually that the clusters of high volatilities decay very slowly, showing a strong tendency of the clustering persistency. In addition, these figures also show that the decay in large volatilities are more monotonic, while the decay in low volatility clusters could be alternating in size. For instance, in the case of the NSDQ return, the lower tail dependence between volatilities of the past 5 days is larger than that of the past 4 days.

In summary, we find a high and durable persistence in the clusters of large volatilities in the both the equity and foreign exchange markets. This is consistent with the long-memory of volatilities documented in the literature. Anderson and Bollerslev (1997) explain the source of the volatility persistence by considering the volatility as a mixture of numerous heterogeneous short-run information arrivals, and the aggregation of these information flow processes may lead to the long memory dependence.

\section{Conclusions}

In this paper, we have analyzed the volatility clustering of a number of return series by using the copula approach with the kernel volatility constructed from the high frequency data. The copula approach has made it possible for us to detect the highly asymmetric pattern in the clusters of high and low volatilities. The availability of the high frequency data, and, thus, the ability to construct the kernel volatility, makes it convenient for us to use the copula approach directly. We found that the volatility clusters in the return series are asymmetric, in the sense that high volatilities tend to cluster more often than low volatilities. 
This asymmetric volatility clusters were visible even when we allowed for the time variation in the volatility clusters. Finally, we found that the clusters are both strongly persistent and durable in the sense that they do not die out even after a one month period. We believe that our findings add to the list of important stylized facts about financial asset returns that are useful for both modeling and forecasting purposes.

\section{References}

[1] Andersen, T. and Bollerslev T. Heterogeneous information arrivals and return volatility dynamics: uncovering the long-run in high frequency returns. The Journal of Finance 1997, VOL. LII, NO. 3, 975-1006.

[2] Andersen, T.G. and Bollerslev, T. Answering the skeptics: yes, standard volatility models do provide accurate forecasts. International Economic Reviews 1998, Vol. 39, $115-158$.

[3] Andersen, T. G., Bollerslev, T. and Diebold, F. X. Roughing it up: Including jump components in the measurement, modeling and forecasting of return volatility. Review of Economics and Statistics 2007, 89, 701-720.

[4] Barndorff-Nielsen, O. E. and Shephard, N. Non-gaussian ornstein-uhlenbeck models and some of their uses in financial economics. The Royal Statistical Society B 2001, Vol. 63, 167-241. 
[5] Bollerslev, T., R. Chou and K. F. Kroner. ARCH modeling in finance. Journal of Econometrics 1992, 52, 5-59.

[6] Bollerslev, T., Litvinova, J. and Tauchen, G. Leverage and volatility feedback effects in high-frequency data. Journal of Financial Econometrics 2006, Vo. 4, No. 3, 353-384.

[7] Boubaker, H. and N. Sghaier, Portfolio optimization in the presence of dependent financial returns with long memory: A copula based approach. Journal of Banking \& Finance 2013, Volume 37, Issue 2, 361-377

[8] Chen, X. and Fan, Y. Estimation of copula-based semiparametric time series models. Journal of Econometrics 2006, 130, 307-335.

[9] Cherubini, U., Luciano, E. and Vecchiato, W. Copula Methods in Finance. John Wiley \& Sons, England; 2004.

[10] Chollete, L., de la Peña, V., Lu, C. International Diversification: A Copula Approach, Journal of Banking and Finance, 2011, 35, pp. 403-417.

[11] Chu, B. Recovering copulas from limited information and an application to asset allocation, Journal of Banking \& Finance, 2011, 35, pp. 1824-1842.

[12] Embrechts, P., McNeil, A. and Strautman, D. Correlation and dependence in risk management: propertites and pitfalls, in Risk Management: Value at Risk and Beyond, ed. M.A.H. Dempster, Cambridge University Press, Cambridge; 2002. 176-223. 
[13] Engle, R. F., Bollerslev T. Modelling the persistence of conditional variances. Econometric Reviews 1986, 5, 1-50, X1-87.

[14] Fermanian, J.-D., Scaillet, O. Some statistical pitfalls in copula modeling for financial applications, in Capital Formation, Governance and Banking, E. Klein (editor), Nova Science Publishing; 2005.

[15] Genest, C., Ghoudi, K., Rivest, L. A semiparametric estimation procedure of dependence parameters in multivariate families of distributions. Biometrika 1995; 82 (3), 543-552.

[16] Hansen, P. R. and Lunde, A. Realized variance and market microstructure noise. Journal of Business and Economic Statistics 2006, Vol. 12, 371-417.

[17] Joe, H. Multivariate models and dependence concepts. Monographs on statistics and applied probability, 73. London: Chapman and Hall; 1997.

[18] Jondeau, E., Rockinger, M. The copula-GARCH model of conditional dependencies: An international stock market application. Journal of International Money and Finance $2006,25,827-853$.

[19] Koopman, S. J., B. Jungbacker and Hol E. Forecasting daily variability of the S\&P 100 stock index using historical realized and implied volatility measurements. Journal of Empirical Finance 2005, Vol 12, 445-475. 
[20] Low, Rand Kwong Yew; Alcockb J.; Faffa R.; Brailsford T. Canonical vine copulas in the context of modern portfolio management: Are they worth it? Journal of Banking \& Finance, 2013, Volume 37, Issue 8, 3085-3099.

[21] Maheu, J. M. and McCurdy, T. H. Do high-frequency measures of volatility improve forecasts of return distribution? Journal of Econometrics 2011, Vol. 160, 69 - 76.

[22] Martens, M. Measuring and forecasting S\&P 500 index futures volatility using highfrequency data. Journal of Future Markets 2002, Vol. 22, 497-518.

[23] McAleer, M., Medeiros M.C. Realized volatility: a review. Econometric Reviews 2008, Vol 27, 10-45.

[24] Nelson, R.B. An introduction to copulas, Springer, New York; 1999.

[25] Ning, C. Dependence Structure Between the Equity Market and the Foreign Exchange Market-A Copula Approach, Journal of International Money and Finance 2010, Vol. 29 Issue 5, 743-759.

[26] Patton, A.L. Modelling asymmetric exchange rate dependence. International Economic Review 2006, 47(2), 527-556.

[27] Patton, A.L. Copula-based models for financial time series, Forthcoming Handbook of Financial Time Series, T. G. Andersen, R. A. Davis, J.-P. Kreiss and T. Mikosch (eds), Springer Verlag; 2008. 
[28] Ray, B. and Tsay, R. Long-range dependence in daily stock volatilities. Journal of Business and Economic Statistics 2000, 18(2),254-262.

[29] Rodriguez, J. C. Measuring financial contagion: a copula approach. Journal of Empirical Finance 2006, 14, 401-423.

[30] Sklar, A. Functions de répartition á n dimensions et leurs marges, Publications de l'Institut de Statistique de l'Université de Paris, 1959, 8, 229-231.

[31] van Dijk, D. and Martens, M. Measuring volatility with the realized range. Journal of Econometrics 2007, 138, 181-207.

[32] Wang, Y. C.; Wu, J. L.; Y. H. Lai. A revisit to the dependence structure between the stock and foreign exchange markets: A dependence-switching copula approach. Journal of Banking \& Finance 2013, Volume 37, Issue 5, 1706-1719

[33] Zhang, L. Mykland, P. A., and Ait-Sahalia, Y. A tale of two scales: determining integrated volatility with noisy high frequency data. Journal of the American Statistical Association 2005, vol. 100, 1394-1411. 
Table 1: Descriptive Statistics of Realized Volatility

\begin{tabular}{cccccccccc}
\hline & SPX & RUT & DJI & IXIC & StoXX50 & GBP & EUR & CHF & JPY \\
\hline Mean & 1.38 & 1.43 & 1.35 & 1.24 & 1.91 & 0.36 & 0.44 & 0.47 & 0.50 \\
Standard deviation & 3.16 & 2.68 & 3.14 & 2.14 & 3.72 & 0.51 & 0.45 & 0.38 & 0.64 \\
Skewness & 12.89 & 9.31 & 12.83 & 9.03 & 12.59 & 6.46 & 6.23 & 4.72 & 8.41 \\
Kurtosis & 298.64 & 149.26 & 287.43 & 139.38 & 290.78 & 58.76 & 72.56 & 41.86 & 126.25 \\
Min & 0.05 & 0.05 & 0.05 & 0.04 & 0.01 & 0.04 & 0.03 & 0.04 & 0.02 \\
Max & 93.13 & 64.25 & 91.26 & 50.09 & 109.22 & 8.70 & 8.86 & 6.42 & 14.68 \\
Count & 2567 & 2567 & 2567 & 2567 & 2592 & 2555 & 2555 & 2555 & 2555 \\
\hline
\end{tabular}


Table 2: Results from Different Copulas

\begin{tabular}{|c|c|c|c|c|c|c|c|c|c|}
\hline & SPX & RUT & DJI & NSDQ & Stoxx 50 & GBP & EUR & $\mathrm{CHF}$ & JPY \\
\hline Linear $\rho$ & 0.64 & 0.67 & 0.65 & 0.68 & 0.58 & 0.88 & 0.66 & 0.65 & 0.70 \\
\hline \multicolumn{10}{|c|}{ Gaussian Copula } \\
\hline$\rho$ & 0.80 & 0.73 & 0.80 & 0.82 & 0.83 & 0.67 & 0.72 & 0.63 & 0.68 \\
\hline Std error & 0.006 & 0.007 & 0.006 & 0.005 & 0.005 & 0.009 & 0.008 & 0.010 & 0.009 \\
\hline AIC & -2614 & -1961 & -2591 & -2886 & -3020 & -1540 & -1831 & -1257 & -1549 \\
\hline $\mathrm{BIC}$ & -2608 & -1955 & -2585 & -2880 & -3015 & -1534 & -1825 & -1251 & -1543 \\
\hline \multicolumn{10}{|l|}{ t Copula } \\
\hline$\rho$ & 0.81 & 0.74 & 0.80 & 0.83 & 0.84 & 0.67 & 0.72 & 0.63 & 0.68 \\
\hline Std error & 0.006 & 0.008 & 0.006 & 0.006 & 0.005 & 0.012 & 0.009 & 0.012 & 0.011 \\
\hline$\nu$ & 9.17 & 7.16 & 6.89 & 8.15 & 51.00 & 3.92 & 5.02 & 6.32 & 5.07 \\
\hline Std error & 1.613 & 1.144 & 1.027 & 1.332 & 0.002 & 0.452 & 0.673 & 0.956 & 0.636 \\
\hline $\mathrm{AIC}$ & -2661 & -2016 & -2665 & -2947 & -3059 & -1656 & -1927 & -1322 & -1652 \\
\hline $\mathrm{BIC}$ & -2649 & -2005 & -2653 & -2936 & -3047 & -1645 & -1915 & -1310 & -1640 \\
\hline symmetric tail & 0.32 & 0.30 & 0.38 & 0.38 & 0.04 & 0.37 & 0.36 & 0.24 & 0.32 \\
\hline \multicolumn{10}{|l|}{ SJC Copula } \\
\hline$\lambda_{L}$ & 0.30 & 0.17 & 0.36 & 0.39 & 0.36 & 0.28 & 0.42 & 0.31 & 0.33 \\
\hline Std error & 0.016 & 0.023 & 0.033 & 0.004 & 0.002 & 0.313 & 0.024 & 0.027 & 0.027 \\
\hline$\lambda_{R}$ & 0.74 & 0.70 & 0.73 & 0.75 & 0.76 & 0.64 & 0.61 & 0.52 & 0.59 \\
\hline Std error & 0.007 & 0.007 & 0.006 & 0.007 & 0.001 & 0.124 & 0.012 & 0.015 & 0.012 \\
\hline $\mathrm{AIC}$ & -2780 & -2275 & -2735 & -2953 & -3071 & -1843 & -1939 & -1326 & -1687 \\
\hline $\mathrm{BIC}$ & -2769 & -2264 & -2723 & -2941 & -3059 & -1831 & -1927 & -1314 & -1676 \\
\hline \multicolumn{10}{|c|}{ Clayton survival Copula } \\
\hline$\lambda_{R}$ & 0.75 & 0.71 & 0.75 & 0.76 & 0.77 & 0.65 & 0.65 & 0.56 & 0.62 \\
\hline Std error & 0.005 & 0.007 & 0.006 & 0.005 & 0.005 & 0.008 & 0.008 & 0.011 & 0.009 \\
\hline $\mathrm{AIC}$ & -2701 & -2241 & -2626 & -2830 & -2991 & -1739 & -1735 & -1207 & -1546 \\
\hline $\mathrm{BIC}$ & -2695 & -2236 & -2620 & -2824 & -2985 & -1733 & -1729 & -1201 & -1540 \\
\hline \multicolumn{10}{|l|}{ Clayton Copula } \\
\hline$\lambda_{L}$ & 0.61 & 0.52 & 0.63 & 0.65 & 0.66 & 0.50 & 0.57 & 0.47 & 0.52 \\
\hline Std error & 0.010 & 0.013 & 0.009 & 0.008 & 0.008 & 0.014 & 0.011 & 0.015 & 0.013 \\
\hline $\mathrm{AIC}$ & -1503 & -1030 & -1562 & -1748 & -1803 & -927 & -1241 & -815 & -1005 \\
\hline $\mathrm{BIC}$ & -1497 & -1024 & -1556 & -1742 & -1797 & -921 & -1236 & -810 & -999 \\
\hline
\end{tabular}




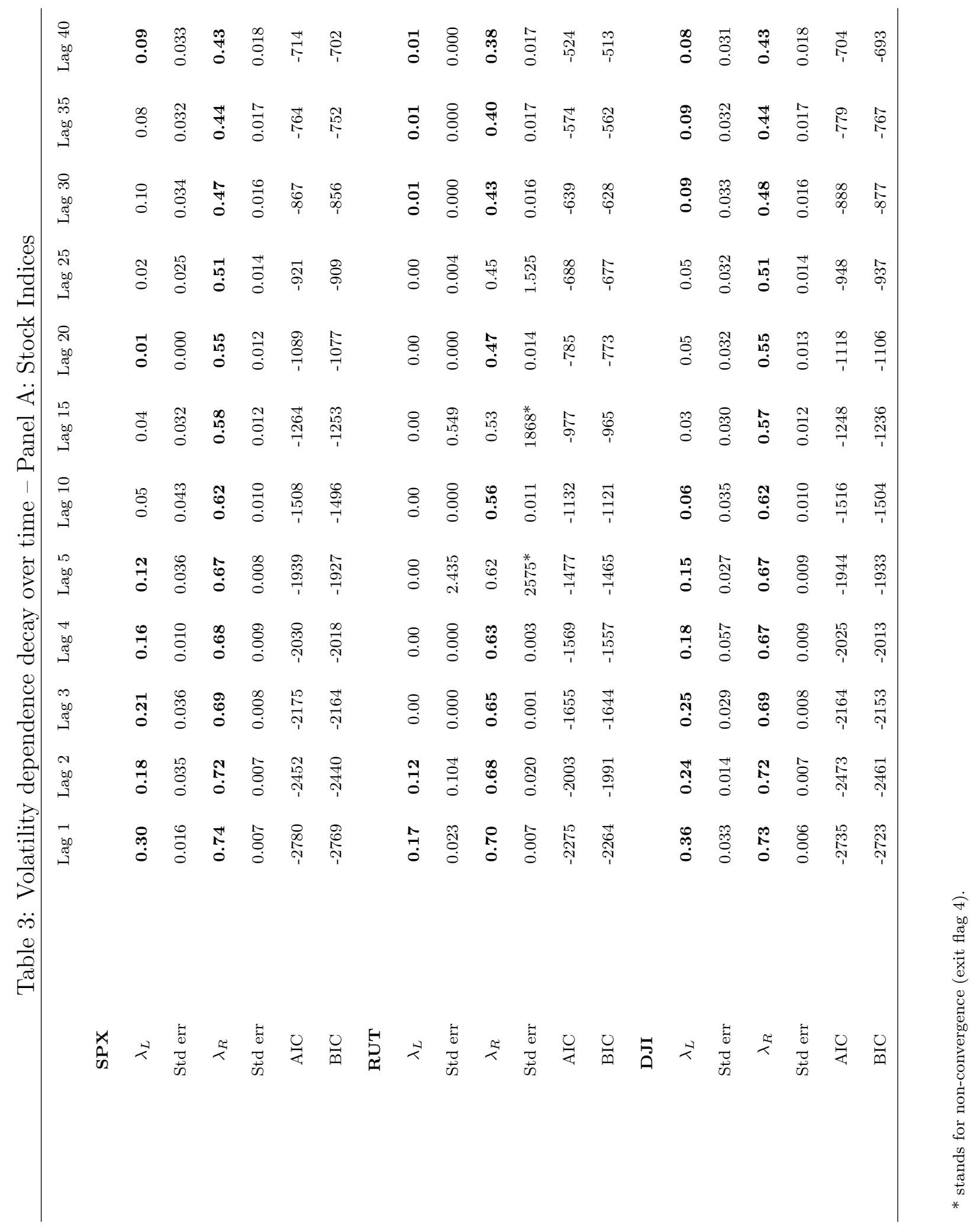




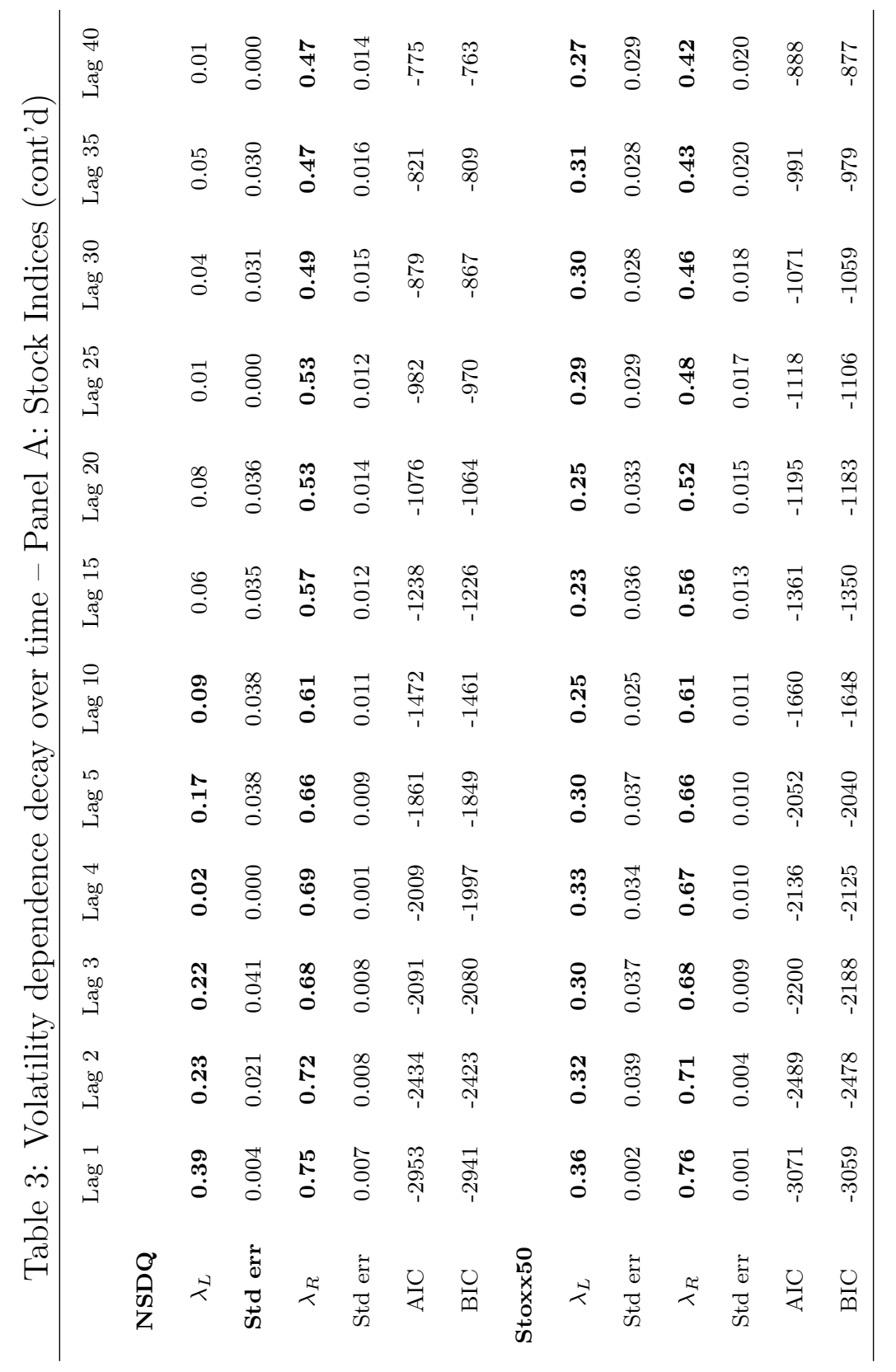




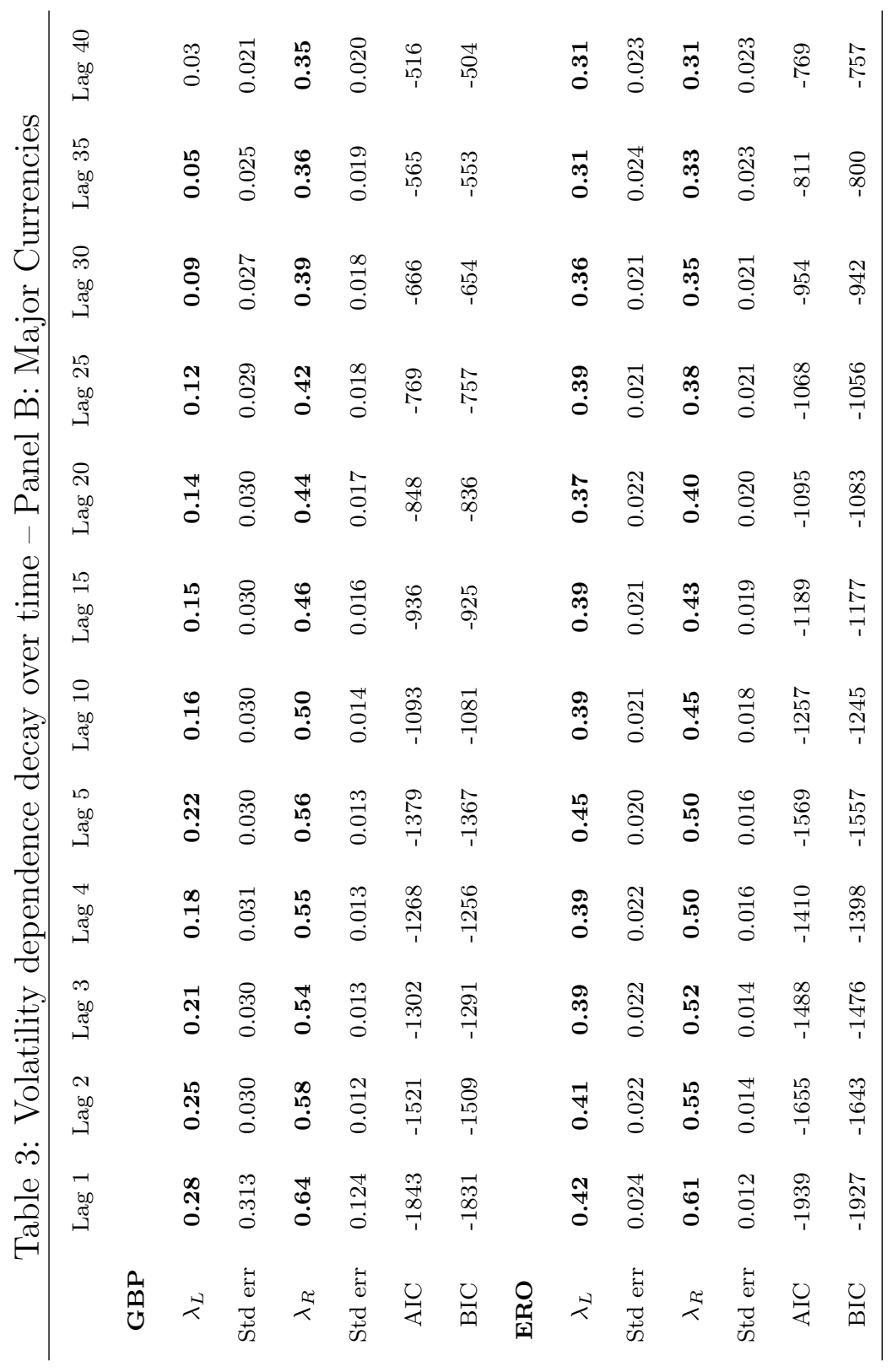




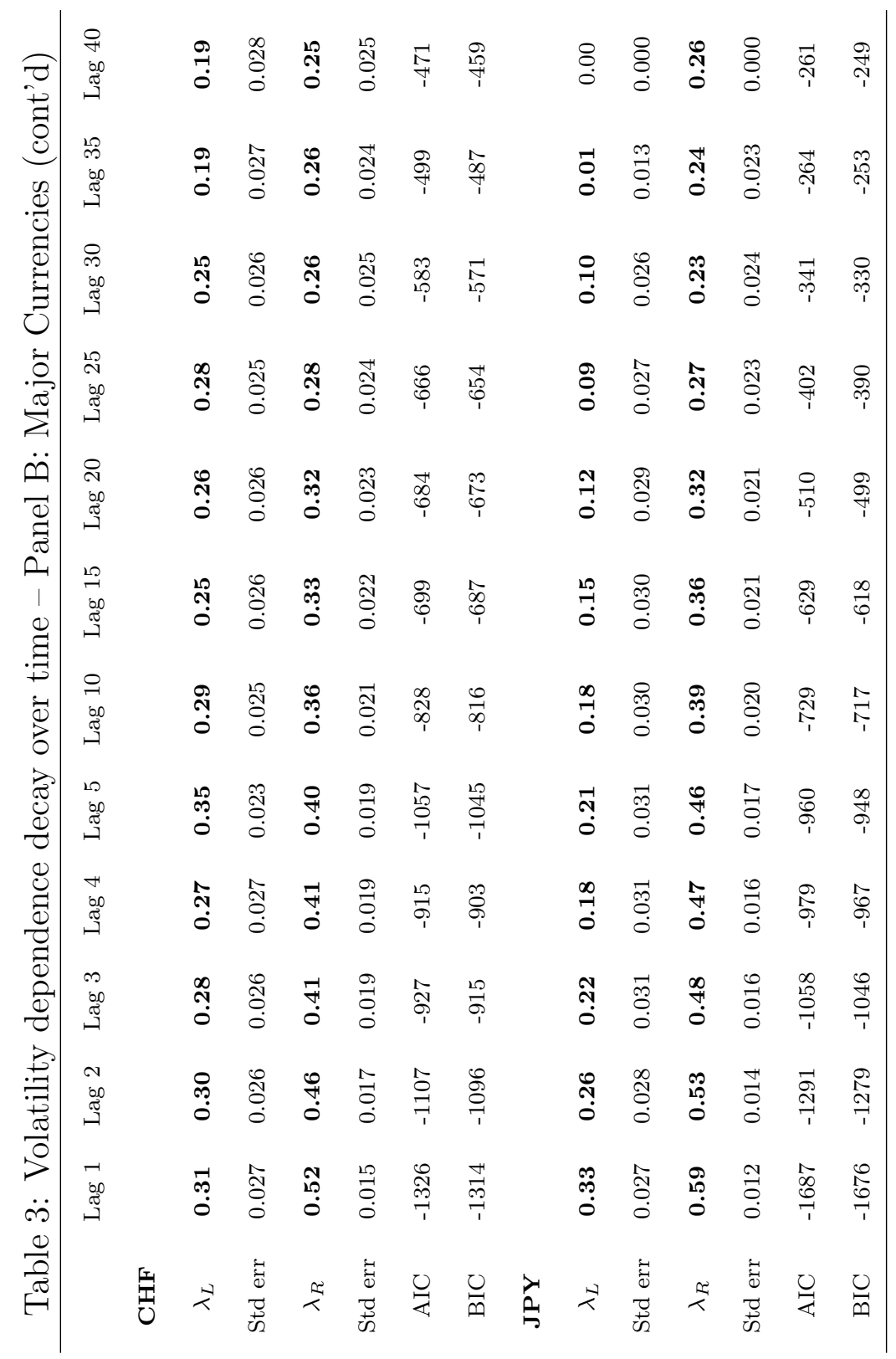


Figure 1a Kernel Volatilities for indices
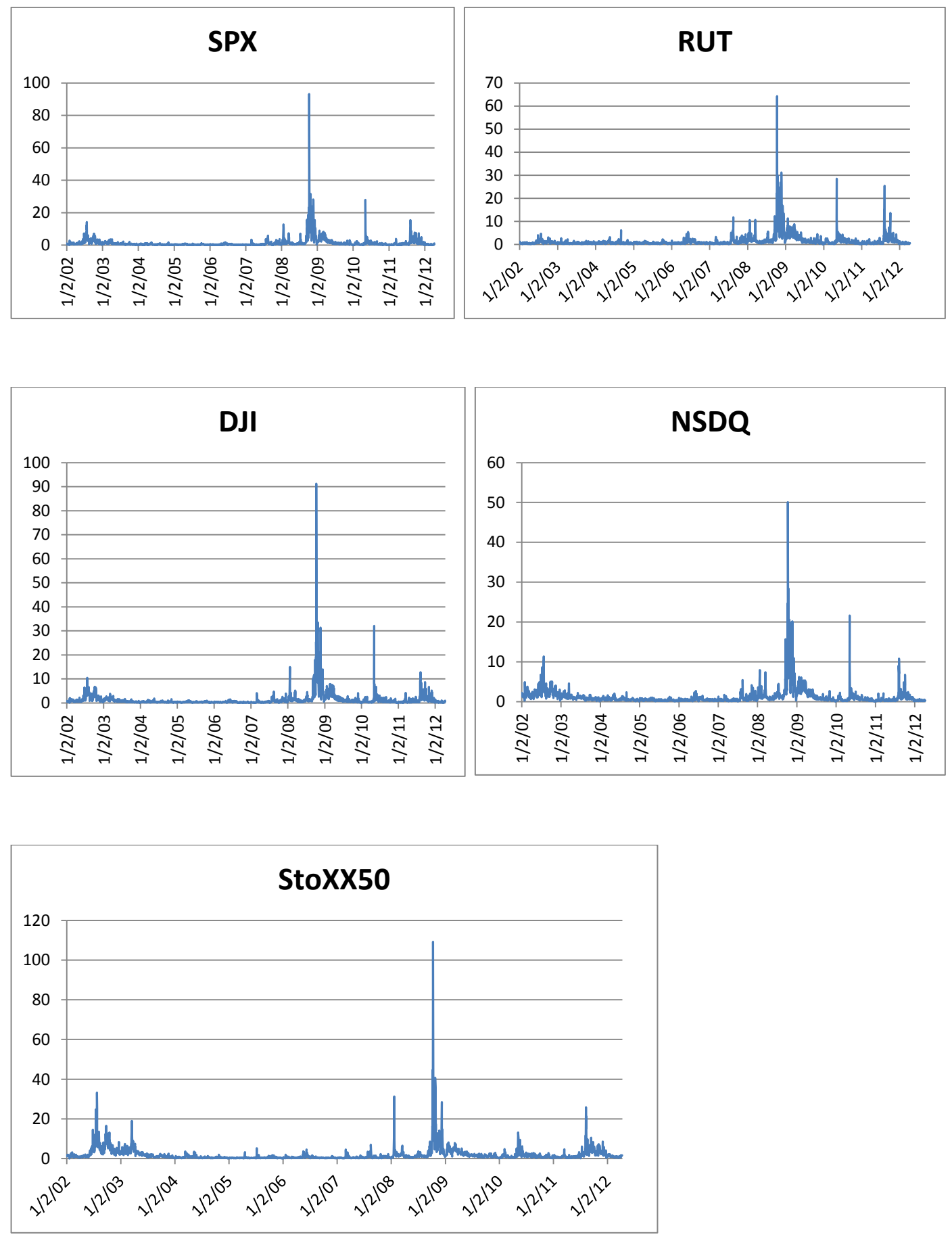
Figure 1b Kernel Volatilities for Exchange Rates
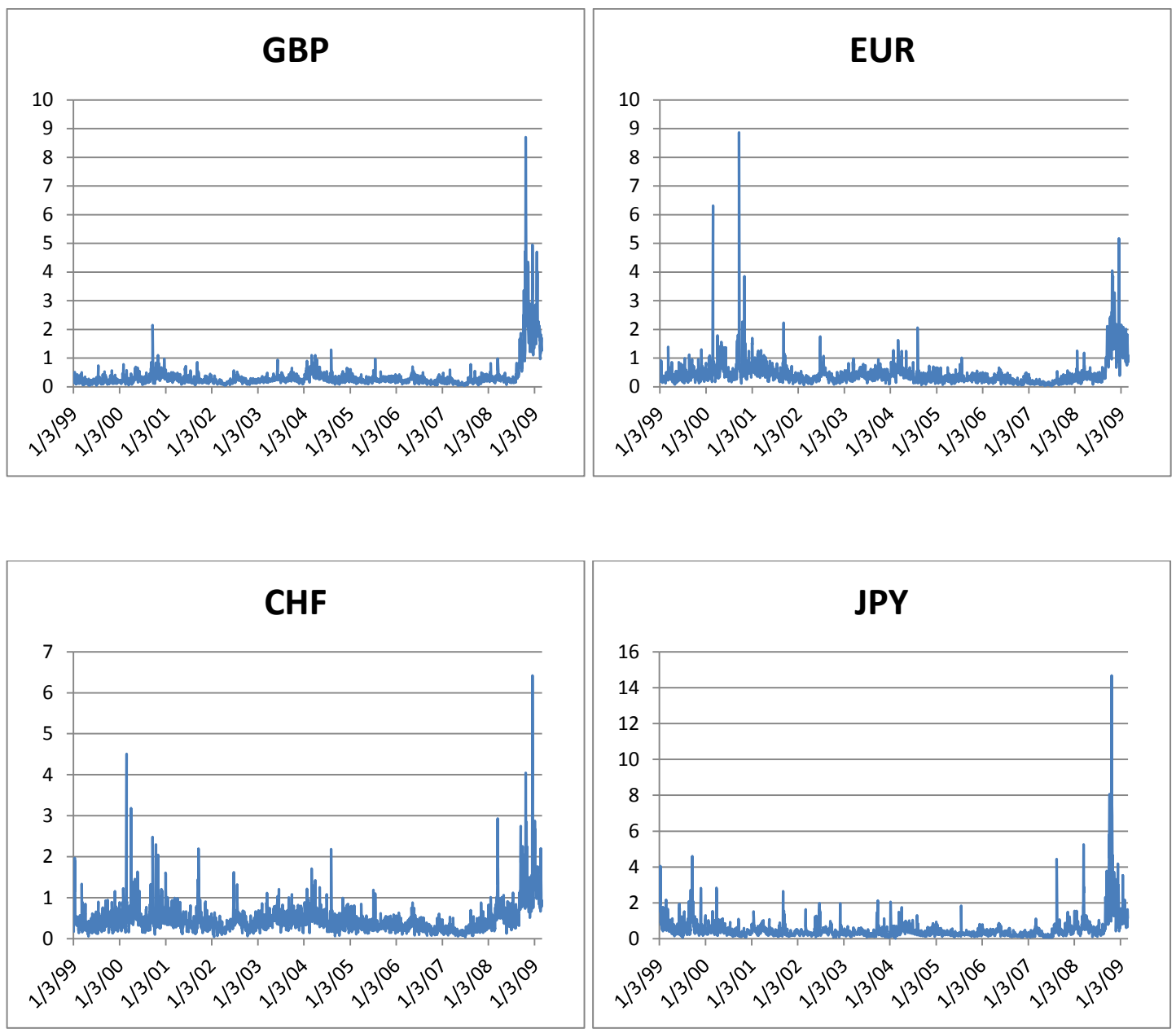
Figure 2. Long memory of high volatility clusters

\section{A. For stock indices}

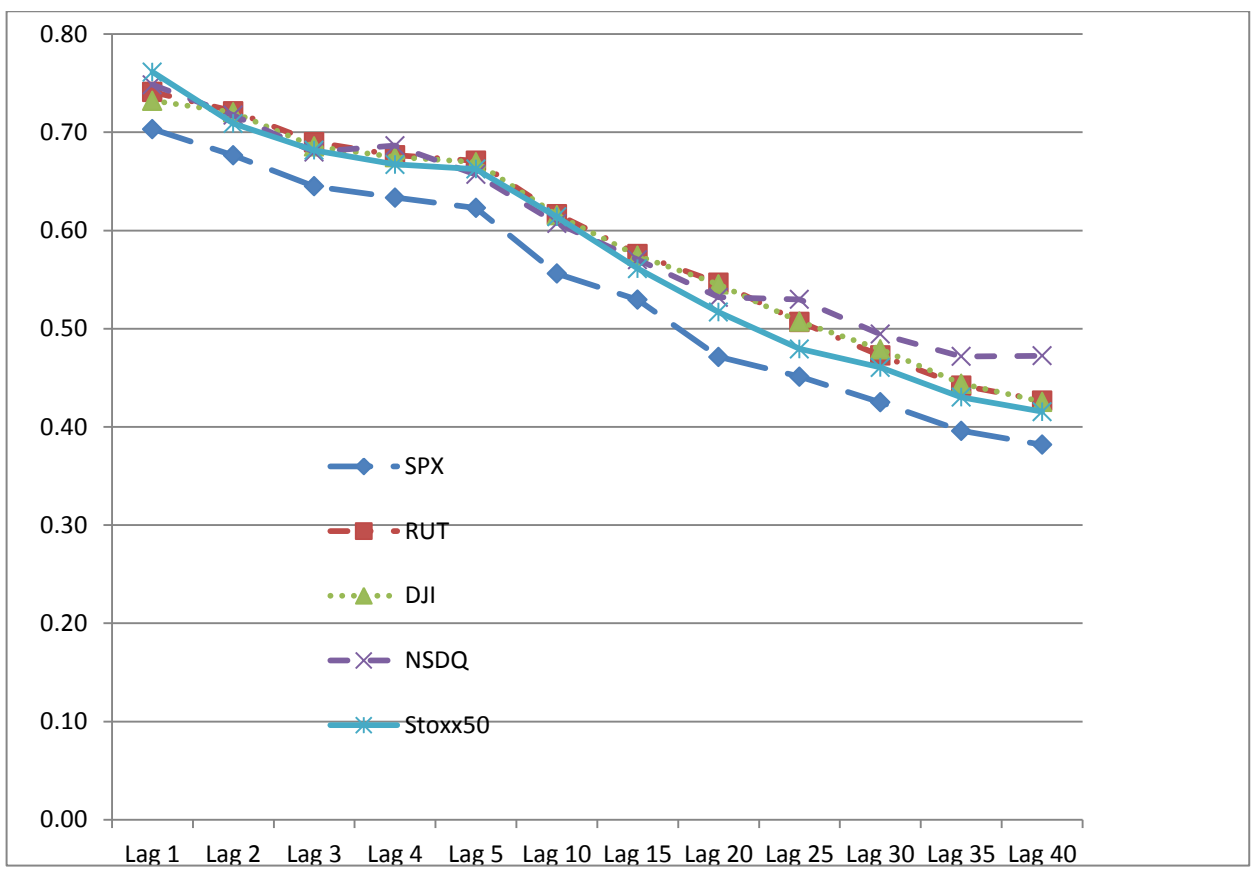

B. For currencies

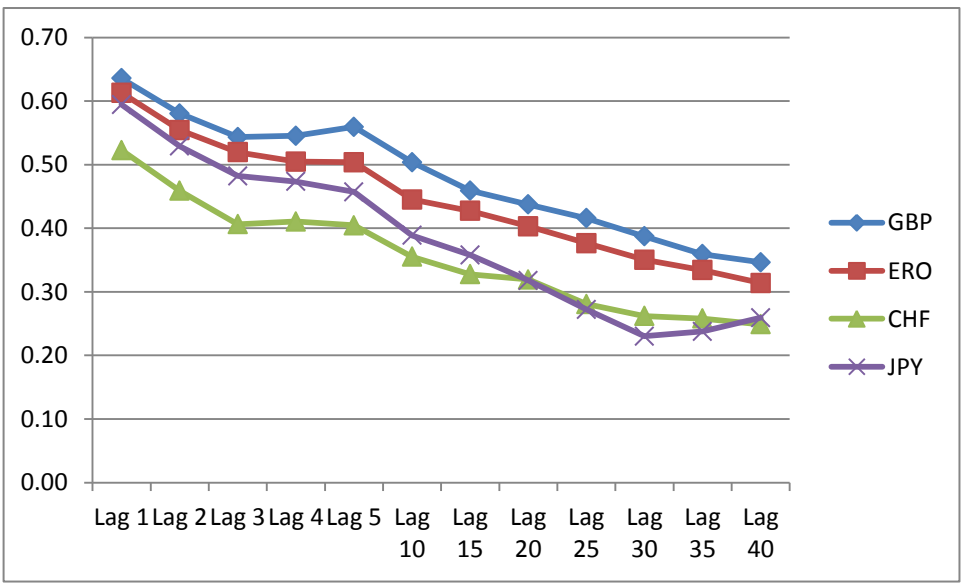


Figure 3. Decay of low volatility clusters

\section{A. For stock indices}

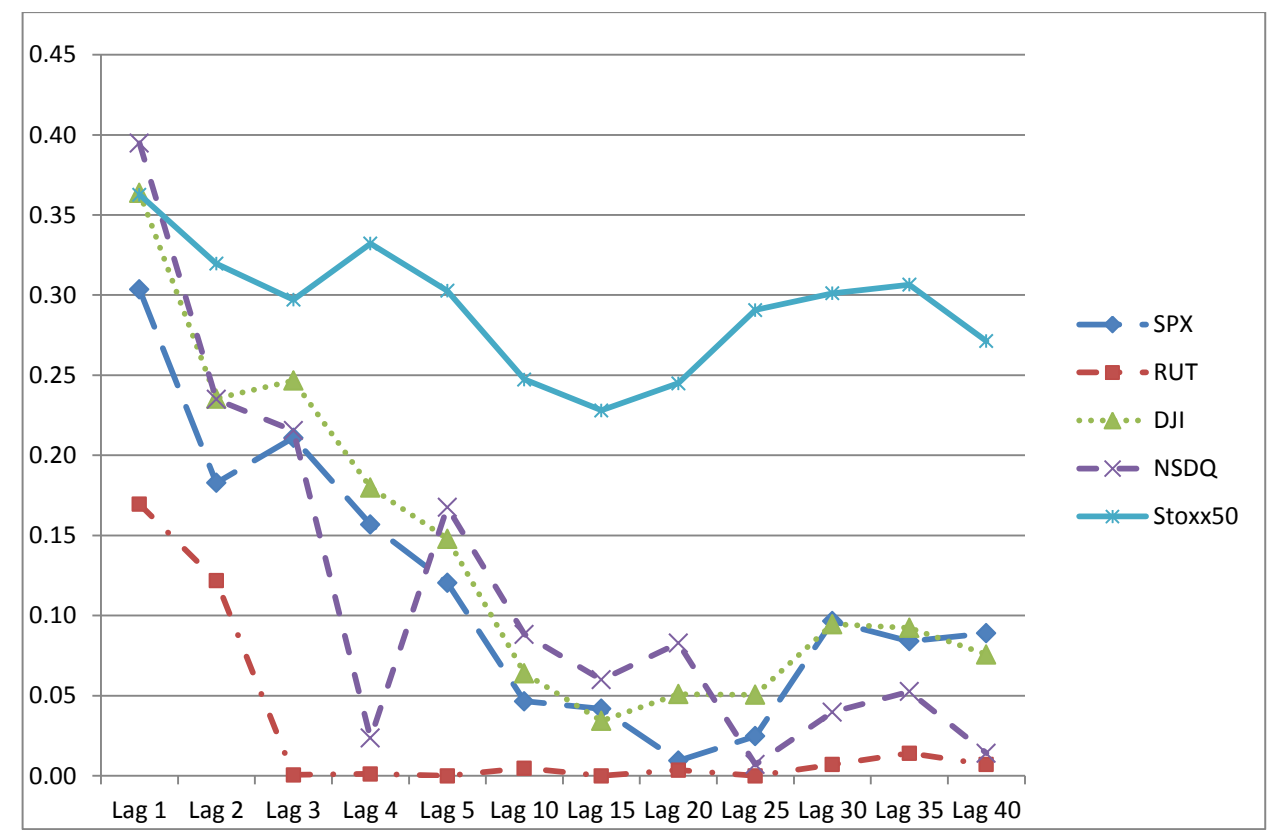

B. For currencies

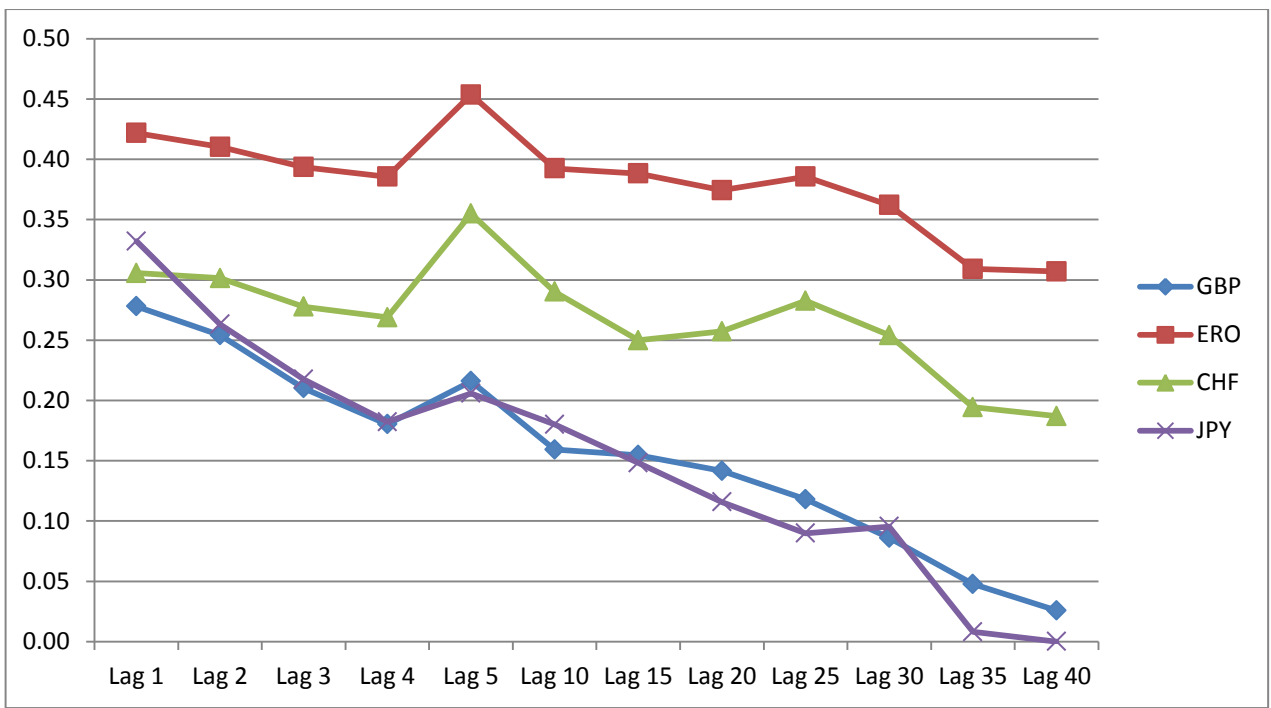

